\title{
High-signal-intensity MR Image in the Hepatobiliary Phase Predicts Long-term Survival in Patients With Hepatocellular Carcinoma
}

\author{
SHUN-ICHI ARIIZUMI ${ }^{1}$, DAISUKE BAN ${ }^{2}$, YUTA ABE ${ }^{3}$, TAKAFUMI KUMAMOTO ${ }^{4}$, \\ SATOSHI KOIZUMI ${ }^{5}$, MINORU TANABE ${ }^{2}$, MASAHIRO SHINODA ${ }^{3}$, \\ ITARU ENDO ${ }^{4}$, TAKEHITO OTSUBO ${ }^{5}$ and MASAKAZU YAMAMOTO ${ }^{1}$ \\ ${ }^{1}$ Department of Surgery, Institute of Gastroenterology, Tokyo Women's Medical University, Tokyo, Japan; \\ ${ }^{2}$ Department of Hepato-Biliary-Pancreatic Surgery, \\ Graduate School of Medicine, Tokyo Medical and Dental University, Tokyo, Japan; \\ ${ }^{3}$ Department of Surgery, School of Medicine, Keio University, Tokyo, Japan; \\ ${ }^{4}$ Department of Gastroenterological Surgery, Yokohama City University, \\ Graduate School of Medicine, Yokohama, Japan; \\ ${ }^{5}$ Division of Gastroenterological and General Surgery, \\ St. Marianna University School of Medicine, Kawasaki, Japan
}

\begin{abstract}
Background/Aim: The aim of the study was to evaluate surgical outcomes of patients with high-signal intensity (SI) image hepatocellular carcinoma (HCC). Patients and Methods: Between 2008 and 2013, 257 HCC patients were retrospectively evaluated. A total of 21 patients were diagnosed as high-SI image HCC, 215 as low-SI image HCC, and 21 patients as mixed (high and low)-SI image $H C C$ in the hepatobiliary (HB) phase of MRI. Five-year overall survival (OS) and recurrence-free survival (RFS) were compared among patient groups. Results: The 5-year $O S$ and RFS rates were significantly higher in patients with high-SI image HCC (100\% and 56\%) than in patients with low-SI image HCC (71\%; $p=0.097$ and $38 \% ; p=0.0209)$ and in patients with mixed-SI image HCC (73\%; $p=0.0329$ and 9\%; $p=0.0021)$. High-SI image was an independent prognostic factor for OS (relative risk 0.167, $p=0.0178$ ) and RFS (relative risk 0.471, $p=0.0322$ ) on multivariate analysis. Conclusion: Patients with high-SI image HCC showed favorable long-term survival after curative surgery.
\end{abstract}

This article is freely accessible online.

Correspondence to: Masakazu Yamamoto, Department of Surgery, Institute of Gastroenterology, Tokyo Women's Medical University, Kawada 8-1, Shinjuku-ku, Tokyo 162-0054, Japan. Tel: +81 333538111, Fax: +81 352697507, e-mail: yamamoto.masakazu@ twmu.ac.jp

Key Words: Hepatocellular carcinoma, Gd-EOB-DTPA-enhanced MRI, high-signal-intensity, surgical outcome.
Gadoxetic acid disodium (Gd-EOB-DTPA) is a newly developed liver-specific magnetic resonance imaging (MRI) contrast agent providing combined dynamic images and hepatobiliary (HB) images. This contrast agent is concentrated in the liver parenchyma in the HB phase and is reported to improve the detection of small hepatocellular carcinomas (HCCs) and metastatic liver cancers (1). GdEOB-DTPA-enhanced MRI is useful to predict the histological differentiation of HCC and microscopic portal vein invasion of HCC preoperatively (2-5).

This contrast agent is taken into hepatocytes via a transporter which is called organic anion transporting polypeptide 8 (OATP8) (6, 7). Therefore, typical HCC commonly shows a low-signal intensity (SI) on the HB images compared with the liver parenchyma because there is no OATP in cancer cells. However, high-SI HCC on HB images has been reported (6-10). The relationship between high SI on HB images and overexpression of OATP8 of HCC cells has been reported $(6,7,9)$. Furthermore, the prognostic impact of high-SI HCC on 3-year survival rate has also been reported $(9,11,12)$. However, long-term surgical outcomes after curative hepatectomy in patients with high-SI HCC have not been evaluated because high-SI HCC is very rare. Therefore, we attempted to clarify whether there might be a difference in long-term surgical outcomes on the basis of the HB phase images of Gd-EOB-DTPA-enhanced MRI.

\section{Patients and Methods}

Between 2008 and 2013, a total of 257 patients with Child-pugh class A and single HCC or 2 nodules HCC were retrospectively 
identified from 5 medical university hospitals. Of those, 21 patients showed uncommon high-SI image in the HB phase on Gd-EOBDTPA-enhanced MRI (Figure 1a and 1b). The patients comprised 16 men $(76 \%)$ and 5 women $(24 \%)$, and the median age was 71 years (range $=55-82$ years). All patients were given a diagnosis of HCC preoperatively because they showed typical HCC images (high density in the arterial phase and low density in the venous phase) on multidetector computed tomography (MDCT) or dynamic MRI. Of the 21 patients, $17(81 \%)$ were given diagnoses of single HCC and $4(19 \%)$ were given diagnoses of two nodular HCCs. The median tumor size on CT scan was $3.5 \mathrm{~cm}($ range $=1-10 \mathrm{~cm})$. No patients showed major vascular invasion of HCC.

To determine the prognostic impact of the high-SI image HCC in the HB phase, 215 patients with typical low-SI image HCC in the HB phase were also evaluated (Figure 1c). All those 215 patients showed Child-Pugh class A, typical HCC images, single or 2 nodules, median tumor size of $3.2 \mathrm{~cm}$, and no major vascular invasion of HCC. The remaining 21 patients with heterogeneous mixed (high and low)-SI image HCC were also evaluated (Figure 1d).

This study was proposed by the association for clinical research on surgery (ACRoS-1401) of 5 medical university hospitals. Information of this study was obtained by opt-out for all patients because this study was a retrospective cohort study. The local committees for medical ethics and clinical studies of each of the 5 medical universities approved this study. The ethics committee of TWMU approved this study (approval number: 3084 ).

Gd-EOB-DTPA-enhanced MRI. MRI was performed according to the protocol of each hospital. All patients received a dose of $0.1 \mathrm{ml} / \mathrm{kg}$ Gd-EOB-DTPA (Primovist; Bayer Schering Pharma AG, Berlin, Germany) intravenously at a rate of $1 \mathrm{ml} / \mathrm{sec}$. Dynamic studies (arterial dominant phase, portal phase, venous phase, and equilibrium phase) and $\mathrm{HB}$ phase images were taken at 15-20 min after the injection (4).

Signal intensity (SI) within the tumor in the HB phase was classified into high-SI, low-SI, or mixed-SI HCC. Tumors that showed a homogenous high-SI within the tumor in the HB phase compared with that of the liver parenchyma and determined to be high-SI image HCC (Figure 1a, b). On the other hand, tumors that showed a homogenous low-SI within the tumor were determined to be low-SI image HCC (Figure 1c). Tumors that showed a heterogeneous high- and low-SI within the tumor were determined mixed-SI image HCC (Figure 1d). The relative SI within the tumor was also assessed as follows; the SI of the tumor and liver parenchyma were measured on the same image using oval regions of interest (ROI). The relative SI was calculated as follows: Relative SI=SI tumor/SI liver parenchyma (9). These findings were determined by all authors who had over 15 years of experience in liver surgery and a radiologist with 20 years of experience in radiological diagnosis.

Surgery and pathological evaluation. All patients underwent hepatectomy within 2 weeks after Gd-EOB-DTPA-enhanced MRI at each of the 5 university hospitals. Sixteen of 21 patients with high-SI HCC underwent anatomical hepatectomy, and 5 patients underwent non-anatomical partial resection. The choice of resection was made on the basis of the tumor location and liver function (ICGR15). No mortality was seen in the 21 patients with high-SI HCC. Macroscopic morphology of tumor type and pathological findings were examined according to the General Rules for the
Clinical and Pathological Study of Primary Liver Cancer of the Liver Cancer Study Group of Japan (13). The terminology of liver resection was determined based on the Terminology Committee of the International Hepato-Pancreato-Biliary Association in 2000 (14).

Follow-up and treatment of patients with recurrence. After surgery, patients were followed up every 4-12 weeks at the 5 university hospitals, and the mean follow-up period was $45 \pm 28$ months (range $=0.7-112$ months). Ultrasonography or CT was performed once every 3-4 months. Cause-specific survival was defined as the time from hepatectomy to the date of cancer-death, liver failuredeath or last contact. When a solitary HCC recurred in the liver, hepatectomy was repeated or radiofrequency ablation was performed. When multiple HCCs recurred in the liver, transarterial chemoembolization (TACE) was performed.

Statistical analysis. Categorical variables were assessed using the chi-square test or Fisher exact test, and continuous variables were assessed using the $t$-test. Continuous variables were divided into two groups according to the median value (Age, ICGR 15, and tumor size) and normal cut off value (AFP and PIVKA-II). Patient overall survival (OS) and recurrence-free survival (RFS) were calculated using the Kaplan-Meier method, and comparison of survival curves was made with the log-rank test. Potential predictors of OS and RFS were evaluated in a multivariate Cox's proportional hazard model. $p$-Values less than $0.05(p<0.05)$ were taken to indicate statistical significance. JMP software (version 13.0; SAS Institute, Cary, NC, USA) was used for statistical analysis.

\section{Results}

Twenty-one of 257 patients showed high-SI image HCC, and the rate of patients with high-SI image $\mathrm{HCC}$ was $8.1 \%$. The patient clinical characteristics are shown in Table I. After comparison between patients with high-SI image HCC and low-SI image HCC, gender did not differ between groups, nor did the median age. The median tumor size did not differ between groups, nor did the number of tumors, and the number of cirrhosis cases. The median ICGR 15, AFP, and PIVKA-II did not differ between groups. The number of patients with anatomical hepatectomy did not differ between groups. Eighteen of 21 patients (86\%) with high-SI image HCC showed the simple nodular (SN) type and 13 of 21 patients $(62 \%)$ showed the so-called green hepatoma. The $\mathrm{SN}$ type and so-called green hepatoma were significantly more frequent in patients with high-SI image HCC than in patients with low-SI image HCC (53\%: $p=0.0035$ and 13\%: $p<0.0001)$. Patients with high-SI image HCC showed well $(33 \%)$ or moderately $(67 \%)$ differentiated HCC. No patients with high-SI image HCC had microscopic portal vein invasion or intrahepatic metastasis. Microscopic portal invasion was significantly lower in patients with high-SI image HCC than in patients with low-SI image HCC (18\%: $p=0.0354)$.

After comparison between patients with high-SI image HCC and those with mixed-SI image HCC, median tumor size did not differ between groups, nor did the number of 


\begin{tabular}{|c|c|c|c|c|c|c|}
\hline & & $\begin{array}{c}\text { High-SI } \\
\text { image } \\
n=21\end{array}$ & $\begin{array}{l}\text { Low-SI } \\
\text { image } \\
n=215\end{array}$ & $\begin{array}{l}\text { Mixed-SI } \\
\text { image } \\
n=21\end{array}$ & $\begin{array}{l}p \text {-Value } \\
\text { High } v s \text {. } \\
\text { Low }\end{array}$ & $\begin{array}{c}p \text {-Value } \\
\text { High } v s . \\
\text { Mixed }\end{array}$ \\
\hline Gender & Male & $16(76 \%)$ & $170(79 \%)$ & $16(76 \%)$ & 0.76 & 0.08 \\
\hline Age & Median (range), year & $71(55-82)$ & $70(35-90)$ & $67(26-80)$ & 0.49 & 0.85 \\
\hline Child-Pugh & A & $21(100 \%)$ & $215(100 \%)$ & $21(100 \%)$ & 1 & 1 \\
\hline Relative signal intensity & Median (range) & $1.22(1.02-1.50)$ & $0.65(0.17-0.99)$ & $0.89(0.55-1.50)$ & $<0.0001$ & $<0.0001$ \\
\hline Tumor size on CT or MRI & Median (range), $\mathrm{cm}$ & $3.5(1-10)$ & $3.2(1-12)$ & $4.2(1.7-17)$ & 0.9 & 0.63 \\
\hline Number of tumors on CT or MRI & Single $/ 2$ nodules & $\begin{array}{c}17(81 \%) / \\
4(19 \%)\end{array}$ & $\begin{array}{l}183(85 \%) / \\
32(15 \%)\end{array}$ & $\begin{array}{c}17(81 \%) / \\
4(19 \%)\end{array}$ & 0.61 & 0.68 \\
\hline Hepatitis C Virus & Present & $7(33 \%)$ & $93(43 \%)$ & $11(52 \%)$ & 0.38 & 1 \\
\hline Cirrhosis & Present & $8(38 \%)$ & $71(33 \%)$ & $9(43 \%)$ & 0.64 & 1 \\
\hline ICGR 15 & Median (range), $\%$ & $14(2-38)$ & $12(3-47)$ & $13(4-43)$ & 0.24 & 0.88 \\
\hline AFP & $\begin{array}{l}\text { Median (range), } \mathrm{ng} / \mathrm{ml} \\
<10 \mathrm{ng} / \mathrm{ml}\end{array}$ & $\begin{array}{l}4(1-739) \\
15(71 \%)\end{array}$ & $\begin{array}{c}10(1-40539) \\
104(48 \%)\end{array}$ & $\begin{array}{l}7(2-810) \\
11(52 \%)\end{array}$ & $\begin{array}{c}0.33 \\
0.0437\end{array}$ & $\begin{array}{l}0.76 \\
0.12\end{array}$ \\
\hline PIVKA-II & $\begin{array}{l}\text { Median (range), U/ml } \\
<40 \mathrm{U} / \mathrm{ml}\end{array}$ & $\begin{array}{c}27(10-7319) \\
14(67 \%)\end{array}$ & $\begin{array}{c}73(10-226200) \\
87(41 \%)\end{array}$ & $\begin{array}{c}192(11-26170) \\
5(24 \%)\end{array}$ & $\begin{array}{c}0.5 \\
0.0238\end{array}$ & $\begin{array}{l}0.41 \\
0.21\end{array}$ \\
\hline Surgical procedure & Anatomical & $16(76 \%)$ & $165(77 \%)$ & $19(90 \%)$ & 0.95 & 0.49 \\
\hline Blood loss & Median (range), $\mathrm{ml}$ & $315(1-2340)$ & $491(1-6342)$ & $684(159-7865)$ & 0.39 & 0.54 \\
\hline Blood transfusion & Present & $2(10 \%)$ & $27(13 \%)$ & $3(14 \%)$ & 0.68 & 0.58 \\
\hline 30 days mortality & Present & 0 & $2(0.9 \%)$ & 0 & 0.99 & 1 \\
\hline Macroscopic tumor type & SN/SNEG or CM & $\begin{array}{c}18(86 \%) / \\
3(14 \%)\end{array}$ & $\begin{array}{l}113(53 \%) / \\
102(47 \%)\end{array}$ & $\begin{array}{l}11(52 \%) / \\
10(48 \%)\end{array}$ & 0.0035 & 0.15 \\
\hline Green hepatoma & Present & $13(62 \%)$ & $29(13 \%)$ & $9(43 \%)$ & $<0.0001$ & 0.0126 \\
\hline Tumor differentiation & Well/Moderately/Poorly-Other & $\begin{array}{c}7(33 \%) / \\
14(67 \%) / \\
0(0 \%)\end{array}$ & $\begin{array}{c}24(11 \%) / \\
159(74 \%) / \\
32(15 \%)\end{array}$ & $\begin{array}{c}2(10 \%) / \\
17(80 \%) / \\
2(10 \%)\end{array}$ & 0.0055 & 0.24 \\
\hline Microscopic portal invasion & Present & $0(0 \%)$ & $38(18 \%)$ & $5(24 \%)$ & 0.0354 & 0.03 \\
\hline Intrahepatic metastasis & Present & $0(0 \%)$ & $18(8 \%)$ & $4(19 \%)$ & 0.17 & 0.15 \\
\hline
\end{tabular}

SI: Signal-intensity; Mixed: High and Low-SI; MRI: magnetic resonance imaging; ICGR15: indocyanine green retention rate at 15 min; AFP: alphafetoprotein; PIVKAII: protein induced by vitamin K absence and antagonist-II; SN: simple nodular type; SNEG: simple nodular with extranodular growth type; CM: confluent multinodular type.

tumors, and the number of cirrhosis cases. The median ICGR15, AFP, and PIVKA-II did not differ between groups, whereas the median relative SI was significantly different between groups (Table I). The green hepatoma was significantly more frequent in patients with high-SI image HCC than in patients with mixed-SI image HCC (43\%: $p=0.0126)$. Microscopic portal invasion was significantly lower in patients with high-SI image HCC than in patients with mixed-SI image HCC (24\%: $p=0.03)$.

No patients with high-SI image HCC died within 30 days after hepatectomy. One patient died of HCC recurrence 88 months after hepatectomy. The overall survival (OS) rate for 21 patients with high-SI image HCC was $100 \%$ at 5 years. The 5-year OS rate was significantly higher in patients with high-SI image HCC $(100 \%)$ than in patients with low-SI image HCC (71\%: $p=0.0297$, Figure 2a). In 7 of 21 patients with high-SI image HCC, HCC recurred in the remnant liver during follow-up. No patients showed extrahepatic recurrence. The 5-year RFS rate was significantly higher in patients with high-SI image HCC (56\%) than in patients with low-SI image HCC (38\%: $p=0.0209$, Figure 2b).
The 5-year OS rate and RFS rate were significantly higher in patients with high-SI image HCC (100\% and 56\%) than in patients with mixed-SI image HCC (73\%: $p=0.0329$, Figure $2 \mathrm{a}$ and $9 \%: p=0.0021$, Figure $2 \mathrm{~b}$ ).

The univariate analysis of prognostic factors of survival is summarized in Table II. The univariate prognostic factors were entered into a multivariate model to identify independent predictors of survival and recurrence free survival. The factors by Cox's proportional hazard model are shown in Table III. High-SI image was a significant independent prognostic factor for OS (relative risk $0.167, p=0.0178$ ) and RFS (relative risk $0.471, p=0.0322$ ) on multivariate analysis. However, mixedSI image was not a significant prognostic factor for OS and RFS. Similarly, relative signal intensity was a significant independent prognositic factor for RFS, but it was not a significant prognostic factor for OS.

\section{Discussion}

Typical HCC commonly showed low-SI in the HB phase compared with the liver parenchyma. However, approximately 

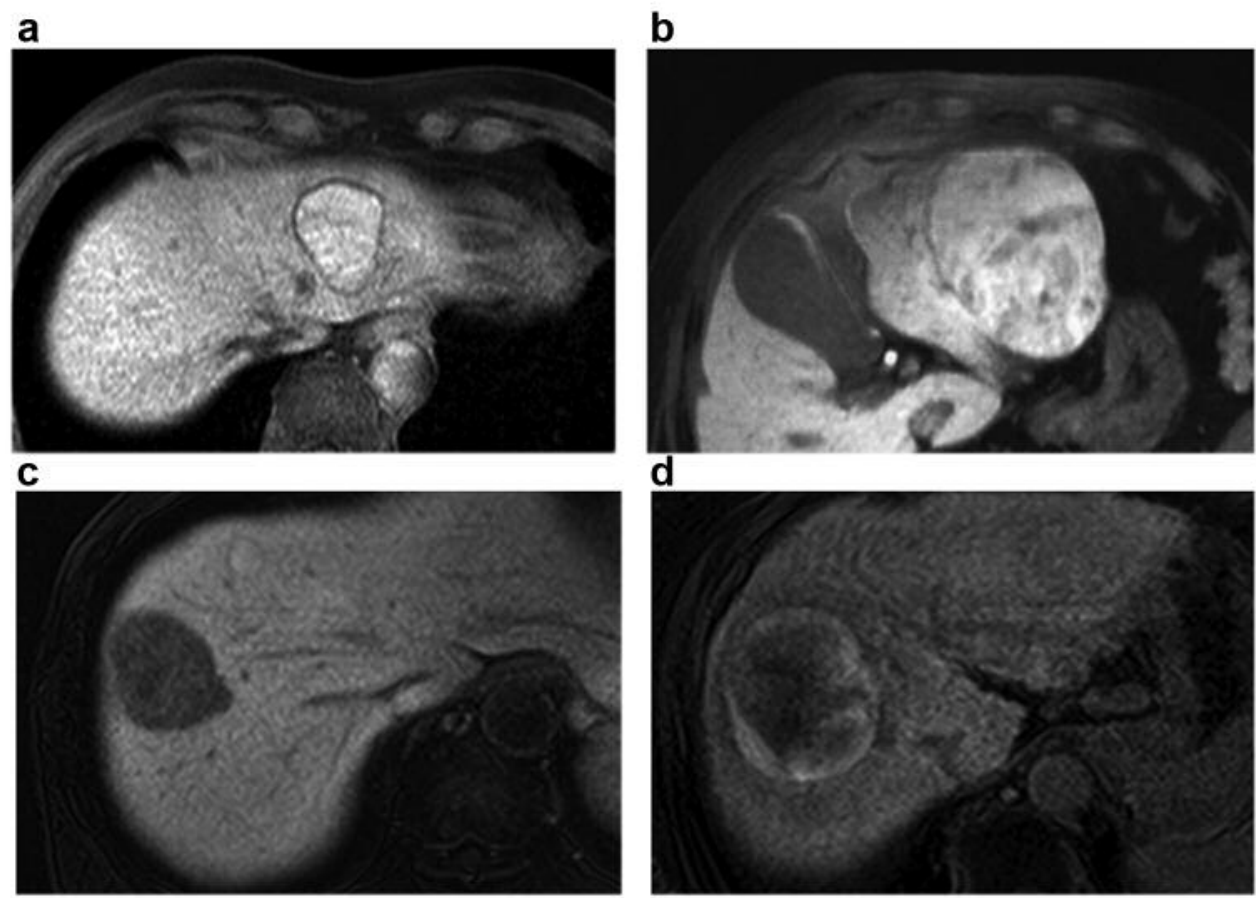

Figure 1. A tumor showing high-signal-intensity (SI) image $(a, b)$, a low-SI image (c), and mixed (high and low)-SI image (d) in the HB phase on Gd-EOB-DTPA-enhanced MRI.

Table II. The univariate analysis of prognostic factors of survival and recurrence-free survival.

\begin{tabular}{|c|c|c|c|c|c|c|}
\hline & & Number & $\begin{array}{c}\text { 5-year } \\
\text { overall } \\
\text { survival }(\%)\end{array}$ & $p$-Value & $\begin{array}{c}\text { 5-year } \\
\text { recurrence-free } \\
\text { survival }(\%)\end{array}$ & $p$-Value \\
\hline \multirow[t]{2}{*}{ Gender } & Male & 203 & $72 \%$ & 0.67 & $36 \%$ & 0.67 \\
\hline & Female & 54 & $79 \%$ & & $40 \%$ & \\
\hline \multirow[t]{2}{*}{ Age, year } & $<70$ & 121 & $83 \%$ & 0.0036 & $47 \%$ & 0.0047 \\
\hline & $\geq 70$ & 136 & $65 \%$ & & $26 \%$ & \\
\hline \multirow{2}{*}{ Hepatitis C Virus } & Absent & 147 & $81 \%$ & 0.0026 & $44 \%$ & 0.0018 \\
\hline & Present & 110 & $64 \%$ & & $26 \%$ & \\
\hline \multirow[t]{2}{*}{ ICGR $15, \%$} & $<15$ & 165 & $83 \%$ & 0.0011 & $43 \%$ & 0.0008 \\
\hline & $\geq 15$ & 92 & $57 \%$ & & $25 \%$ & \\
\hline \multirow[t]{2}{*}{$\mathrm{AFP}, \mathrm{ng} / \mathrm{ml}$} & $<10$ & 130 & $74 \%$ & 0.23 & $38 \%$ & 0.14 \\
\hline & $\geq 10$ & 127 & $73 \%$ & & $35 \%$ & \\
\hline \multirow[t]{2}{*}{ PIVKA-II, U/ml } & $<40$ & 103 & $83 \%$ & 0.0171 & $52 \%$ & $<0.0001$ \\
\hline & $\geq 40$ & 151 & $67 \%$ & & $26 \%$ & \\
\hline \multirow[t]{2}{*}{ Size, $\mathrm{cm}$} & $<3$ & 120 & $84 \%$ & 0.0212 & $38 \%$ & 0.22 \\
\hline & $\geq 3$ & 137 & $65 \%$ & & $36 \%$ & \\
\hline \multirow[t]{2}{*}{ Number } & 1 nodule & 218 & $76 \%$ & 0.09 & $38 \%$ & 0.06 \\
\hline & 2 nodules & 39 & $63 \%$ & & $26 \%$ & \\
\hline \multirow[t]{3}{*}{ Hepatobiliary phase image } & High-SI & 21 & $100 \%$ & High vs. Low: 0.0297 & $56 \%$ & High vs. Low: 0.0209 \\
\hline & Low-SI & 215 & $71 \%$ & High vs. Mixed: 0.0329 & $38 \%$ & High vs. Mixed: 0.0021 \\
\hline & Mixed-SI & 21 & $73 \%$ & & $9 \%$ & \\
\hline \multirow{2}{*}{ Relative signal intensity } & $\mathrm{SI}>1$ & 26 & $96 \%$ & 0.09 & $47 \%$ & 0.0322 \\
\hline & $\mathrm{SI}<1$ & 231 & $71 \%$ & & $35 \%$ & \\
\hline \multirow[t]{2}{*}{ Surgery } & Anatomical & 199 & $77 \%$ & 0.06 & $40 \%$ & 0.15 \\
\hline & No anatomical & 58 & $62 \%$ & & $24 \%$ & \\
\hline
\end{tabular}

ICGR15: Indocyanine green retention rate at $15 \mathrm{~min}$; AFP: alpha-fetoprotein; PIVKAII: protein induced by vitamin K absence and antagonist-II; SI: signal-intensity. 


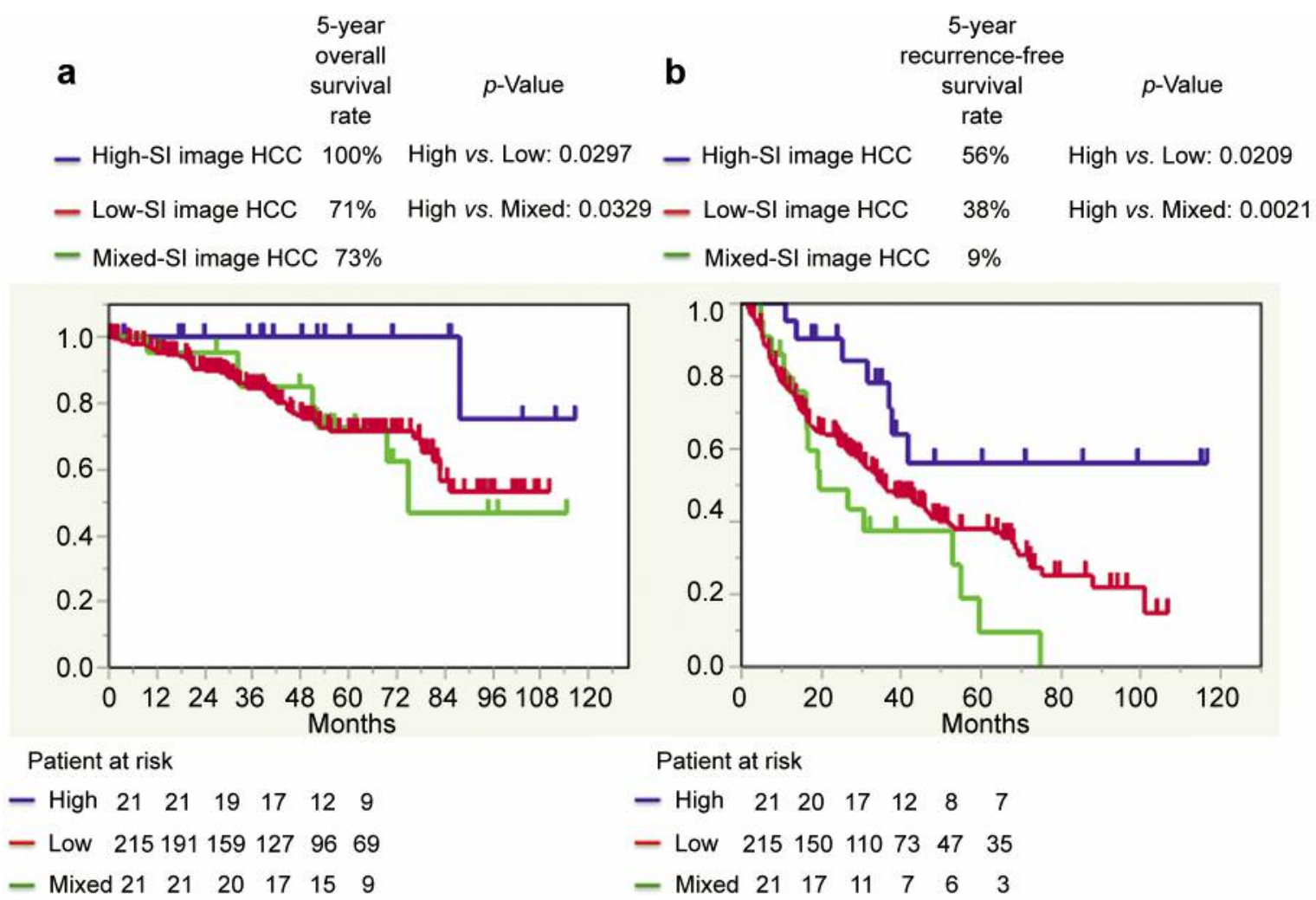

Figure 2. The overall survival curves (a) and recurrence-free survival curves $(b)$.

Table III. Multivariate analysis.

\begin{tabular}{|c|c|c|c|c|c|c|c|}
\hline & & \multicolumn{3}{|c|}{ Overall survival } & \multicolumn{3}{|c|}{ Recurrence-free survival } \\
\hline & & Relative risk & $95 \% \mathrm{CI}$ & $p$-Value & Relative risk & $95 \% \mathrm{CI}$ & $p$-Value \\
\hline \multirow[t]{2}{*}{ Age } & $<70$ & 0.535 & $(0.293,0.949)$ & 0.0321 & 0.747 & $(0.520,1.066)$ & 0.11 \\
\hline & $\geq 70$ & 1 & & & 1 & & \\
\hline \multirow[t]{2}{*}{ Hepatitis C Virus } & Absent & 0.471 & $(0.271,0.809)$ & 0.0064 & 0.602 & $(0.425,0.851)$ & 0.0044 \\
\hline & Present & 1 & & & 1 & & \\
\hline \multirow[t]{2}{*}{ ICGR $15, \%$} & $<15$ & 0.415 & $(0.244,0.701)$ & 0.0011 & 0.630 & $(0.445,0.884)$ & 0.0094 \\
\hline & $\geq 15$ & 1 & & & 1 & & \\
\hline \multirow[t]{2}{*}{ PIVKA-II, U/ml } & $<40$ & 0.849 & $(0.443,1.569)$ & 0.61 & 0.513 & $(0.341,0.733)$ & 0.0005 \\
\hline & $\geq 40$ & 1 & & & 1 & & \\
\hline \multirow[t]{2}{*}{ Size, $\mathrm{cm}$} & $<3$ & 0.431 & $(0.231,0.781)$ & 0.0052 & & & \\
\hline & $\geq 3$ & 1 & & & & & \\
\hline \multirow[t]{3}{*}{ Hepatobiliary phase image } & High-SI & 0.167 & $(0.009,0.782)$ & 0.0178 & 0.471 & $(0.198,0.943)$ & 0.0322 \\
\hline & Mixed-SI & 0.751 & $(0.298,1.644)$ & 0.49 & 1.077 & $(0.597,1.829)$ & 0.79 \\
\hline & Low-SI & 1 & & & 1 & & \\
\hline \multirow[t]{2}{*}{ Relative signal intensity } & SI $>1$ & 0.431 & $(0.104,1.198)$ & 0.12 & 0.534 & $(0.261,0.969)$ & 0.0383 \\
\hline & $\mathrm{SI}<1$ & 1 & & & 1 & & \\
\hline
\end{tabular}

ICGR15: Indocyanine green retention rate at $15 \mathrm{~min}$; PIVKAII: protein induced by vitamin $\mathrm{K}$ absence and antagonist-II; SI: signal-intensity; CI: confidence interval.

$10 \%$ of HCC has been reported to show uncommonly high-SI in the HB phase (6-10). Recently, the 3-year survival rates of patients with high-SI HCC have been reported. Kitao et al. have reported that 22 patients with hyperintense $\mathrm{HCC}$ tended to show longer survival than those with hypointense HCC, and the 3-year survival rate of 22 patients with hyperintense $\mathrm{HCC}$ was $100 \%$ (9). Miura et al. have also reported that in 14 patients with high-SI HCC the 3-year OS rate was $100 \%$ (11). 
In the present study, the OS rate of patients with high-SI image HCC after curative surgery was $100 \%$ at 5 years, because no patients died due to HCC recurrence within 5 years. Moreover, multivariate analysis showed high-SI image HCC to be a significant independent prognostic factor for overall survival (relative risk $=0.167, p=0.0178$ ) and recurrence-free survival (relative risk $=0.471, p=0.0322$ ) in patients with HCC. High-SI image HCC shows uncommon MR images, but patients with high-SI image HCC showed favorable long-term survival after curative surgery.

High-SI HCC is known to show lower levels of serum AFP or PIVKA-II (7-12). Yamashita et al. have reported that Gd-EOB-DTPA uptake in the HB phase correlated with low serum AFP levels, maintenance of hepatocyte function with the up-regulation of OATP1B3 and HNF4A expression, and good prognosis (12). Kitao et al. have reported that the rate of portal vein invasion was significantly lower in patients with hyperintense HCC $(13.6 \%)$ than in patients with hypointense HCCs (36.7\%) (9). Kim et al. have also reported that the rate of microvascular invasion was significantly lower in 18 patients with hyperintense HCCs $(27.8 \%)$ than in patients with hypointense HCCs $(53.5 \%)$ (10). In the present study, 15 of 21 patients with high-SI image HCC showed normal AFP levels $(<10 \mathrm{ng} / \mathrm{ml})$, and no patient with microscopic portal vein invasion of HCC was observed. Moreover, the 5-year RFS rate was significantly higher in patients with high-SI image HCC $(56 \%)$ than in patients with low-SI image HCC (38\%; $p=0.0209)$. Therefore, patients with high-SI image HCC had better surgical outcomes because high-SI image HCC showed less portal vein invasion, less intrahepatic metastasis, and lower recurrence of HCC.

In the present study, 21 patients showed heterogeneous high and low-SI (mixed-SI) image HCC in the HB phase. The patients with heterogeneous mixed-SI image HCC included 5 patients with relative $\mathrm{SI}>1$ and 16 patients with relative $\mathrm{SI}<1$. There have been no previous reports regarding surgical outcome in patients with heterogeneous mixed-SI image HCC. In our present study, 5-year OS rates and RFS rates were significantly higher in patients with high-SI image HCC (100\% and 56\%) than in patients with mixed-SI image HCC (73\%: $p=0.0329$ and 9\%: $p=0.0021$ ). Patients with mixed-SI image HCC showed a heterogeneous high- and low-SI within the tumor but poor surgical outcomes compared with patients with homogeneous high-SI image HCC because some patients with heterogeneous mixed-SI image HCC had microscopic portal invasion and intrahepatic metastasis.

Previous reports have shown better surgical outcomes in patients with hyperintense HCC, and they have shown 3-year OS rates and 3-year RFS rates in relation to the relative SI (between patients with $\mathrm{SI}>1$ and $\mathrm{SI}<1$ ) by univariate analysis $(9,11,12,15)$. Our results showed long-term surgical outcomes (5-year OS rate and 5-year RFS rate) after hepatectomy in relation to the HB image, and high-SI image $\mathrm{HCC}$ revealed as a novel prognostic factor on univariate and multivariate analysis. Furthermore, patients with heterogeneous mixed-SI image HCC showed a high- and lowSI within the tumor but poor surgical outcomes compared with patients with high-SI image HCC. Furthermore, relative SI was not a significant prognostic factor for OS. In conclusion, high-SI image in the $\mathrm{HB}$ phase is a novel prognostic factor for OS and RFS in patients with HCC.

\section{Authors' Contributions}

All Authors participated in conferences to plan and discuss for this manuscript. SA, DB, YA, TK, and SK: treated, operated and collected the date. MT, MS, IE, TO and MY: operated patients. All Authors contributed and agreed with the content of the manuscript.

\section{Conflicts of Interest}

The Authors declare no conflicts of interest regarding this study.

\section{Acknowledgements}

The Authors are indebted to Associate Professor Raoul Breugelmans of the Department of Medical Education of Tokyo Medical University for his review of this manuscript. The authors are indebted to Dr. Ken Morita of the Department of Diagnostic Imaging and Nuclear Medicine of Tokyo Women's Medical University for his help to diagnose of high-or low-SI HCC.

\section{References}

1 Ichikawa T, Saito K, Yoshioka N, Tanimoto A, Gokan T, Takehara Y, Kamura T, Gabata T, Murakami T, Ito K, Hirohashi S, Nishie A, Saito Y, Onaya H, Kuwatsuru R, Morimoto A, Ueda K, Kurauchi $\mathrm{M}$ and Breuer J: Detection and characterization of focal liver lesions. A Japanese phase III, multicenter comparison between Gadoxetic acid disodium-enhanced magnetic resonance imaging and contrast-enhanced computed tomography predominantly in patients with hepatocellular carcinoma and chronic liver disease. Invest Radiol 45: 133-141, 2010. PMID: 20098330. DOI: 10.1097/RLI.0b013e3181caea5b

2 Saito K, Kotake F, Ito N, Ozuki T, Mikami R, Abe K and Shimazaki Y: Gd-EOB-DTPA enhanced MRI for hepatocellular carcinoma: quantitative evaluation of tumor enhancement in hepatobiliary phase. Magn Reson Med Sci 4: 1-9, 2005. PMID: 16127248. DOI: $10.2463 / \mathrm{mrms} .4 .1$

3 Kogita S, Imai Y, Okada M, Kim T, Onishi H, Takamura M, Fukuda K, Igura T, Sawai Y, Morimoto O, Hori M, Nagano H, Wakasa K, Hayashi $\mathrm{N}$ and Murakami T: Gd-EOB-DTPA enhanced magnetic resonance images of hepatocellular carcinoma: correlation with histological grading and portal blood flow. Eur Radiol 20: 2405-2413, 2010. PMID: 20490505. DOI: 10.1007/s00330-010-1812-9

4 Ariizumi S, Kitagawa K, Kotera Y, Takahashi Y, Katagiri S, Kuwatsuru R and Yamamoto M: A non-smooth tumor margin in the hepatobiliary phase of gadoxetic acid disodium (Gd-EOBDTPA)-enhanced magnetic resonance imaging predicts 
microscopic portal vein invasion, intrahepatic metastasis, and early recurrence after hepatectomy in patients with hepatocellular carcinoma. J Hepatobiliary Pancreat Sci 18: 575-585, 2011. PMID: 21360083. DOI: 10.1007/s00534-010-0369-y

5 Kim KA, Kim MJ, Jeon HM, Kim KS, Choi JS, Ahn SH, Cha SJ and Chung YE: Prediction of microvascular invasion of hepatocellular carcinoma: usefulness of peritumoral hypointensity seen on gadoxetate disodium-enhanced hepatobiliary phase images. J Magn Reson Imaging 35: 629-634, 2012. PMID: 22069244. DOI: $10.1002 /$ jmri.22876

6 Narita M, Hatano E, Arizono S, Miyagawa-Hayashino A, Isoda H, Kitamura K, Taura K, Yasuchika K, Nitta T, Ikai I and Uemoto S: Expression of OATP1B3 determines uptake of GdEOB-DTPA in hepatocellular carcinoma. J Gastroenterol 44: 793-798, 2009. PMID: 19404563. DOI: 10.1007/s00535-0090056-4

7 Kitao A, Zen Y, Matsui O, Gabata T, Kobayashi S, Koda W, Kozawa K, Yoneda N, Yamashita T, Kaneko S and Nakanuma Y: Hepatocellular carcinoma: signal intensity at gadoxetic acidenhanced MR Imaging - correlation with molecular transporters and histopathologic features. Radiology 256: 817-826, 2010. PMID: 20663969. DOI: 10.1148/radiol. 10092214

8 Asayama Y, Tajima T, Nishie A, Ishigami K, Kakihara D, Nakayama T, Okamoto D, Fujita N, Aishima S, Shirabe K and Honda $\mathrm{H}$ : Uptake of Gd-EOB-DTPA by hepatocellular carcinoma: radiologic-pathologic correlation with special reference to bile production. Eur J Radiol 80: 243-248, 2011. PMID: 21109378. DOI: 10.1016/j.ejrad.2010.10.032

9 Kitao A, Matsui O, Yoneda N, Kozaka K, Kobayashi S, Koda W, Gabata T, Yamashita T, Kaneko S, Nakanuma Y, Kita R and Arii S: Hypervascular hepatocellular carcinoma: Correlation between biologic features and signal intensity on gadoxetic acidenhanced MR images. Radiology 265: 780-789, 2012. PMID: 23175543. DOI: $10.1148 /$ radiol.12120226

$10 \mathrm{Kim}$ JY, Kim MJ, Kim KA, Jeong HT and Park YN: Hyperintense HCC on hepatobiliary phase images of gadoxetic acid-enhanced MRI: correlation with clinical and pathological features. Eur J Radiol 81: 3877-3882, 2012. PMID: 22954410. DOI: $10.1016 /$ j.ejrad.1012.07.021
11 Miura T, Ban D, Tanaka S, Mogushi K, Kudo A, Matsumura S, Mitsunori Y, Ochiai T, Tanaka $\mathrm{H}$ and Tanabe M: Distinct clinicopathological phenotype of hepatocellular carcinoma with ethoxybenzyl-magnetic resonance imaging hyperintensity: association with gene expression signature. Am J Surg 210: 561569, 2015. PMID: 26105803. DOI: 10.1016/j.ejrad.1012.07.021

12 Yamashita T, Kitao A, Matsui O, Hayashi T, Nio K, Kondo M, Ohno N, Miyati T, Okada H, Yamashita T, Mizukoshi E,Honda M, Nakanuma Y, Takamura H, Ohta T, Nakamoto Y, Yamamoto M, Takayama T, Arii S, Wang X and Kaneko S: Gd-EOB-DTPAenhanced magnetic resonance imaging and alpha-fetoprotein predict prognosis of early-stage hepatocellular carcinoma. Hepatology 60: 1674-1685, 2015. PMID: 24700365. DOI: 10.1002/hep.27093

13 Liver Cancer Study Group of Japan: The General Rules for the Clinical and Pathological Study of Primary Liver Cancer. 2nd English ed. Tokyo, Japan: Kanehara \& Co., Ltd., 2003

14 Terminology committee of the International Hepato-PancreatoBiliary Association: Modified from the Brisbane 2000 Terminology of liver anatomy and resections. HPB 2: 333-339, 2000.

15 Choi JW, Lee JM, Kim SJ, Yoon JH, Baek JH, Han JK and Choi BI: Hepatocellular carcinoma: imaging patterns on gadoxetic acid-enhanced MR Images and their value as an imaging biomarker. Radiology 67: 776-786, 2013. PMID: 23401584. DOI: $10.1148 /$ radiol.13120775
Received May 16, 2019

Revised June 17, 2019

Accepted June 19, 2019 